\title{
LARGER CIRCULAR COLLIDERS
}

E. KEIL

CERN, Geneva, Switzerland

\begin{abstract}
Two types of circular colliders are discussed, muon colliders, and hadron colliders. In both cases, typical values of the most relevant parameters are presented, the state of component development is discussed, schedules for the earliest dates of construction are shown, and critical issues needing R\&D are mentioned.
\end{abstract}

\section{Introduction}

In this talk, I shall restrict myself to high-energy colliders, in particular linear $\mathrm{e}^{+} \mathrm{e}^{-}$ colliders, muon colliders, and hadron colliders. I discuss them in Chapters, 2 and 3, respectively.

\section{Muon Colliders}

The possibility of muon colliders was introduced by Budker ${ }^{1}$, Parkhomchuk and Skrinsky ${ }^{2}$, and Neuffer ${ }^{3}$. It has been developed intensively over the past three years $4,5,6,7,8$. A feasibility study for a $4 \mathrm{TeV}$ muon collider was presented at Snowmass ${ }^{9}$, and a formal collaboration was set up recently ${ }^{10}$.

\subsection{Muon Collider Components}

Fig. 1 shows a schematic layout of a $\mu^{+} \mu^{-}$collider complex. A powerful proton synchrotron accelerates a few short proton bunches that hit a small target for copious $\pi$ production. A system of solenoids and RF cavities captures and accelerates the $\pi$ 's and $\mu$ 's. An ionisation cooling channel reduces the six-dimensional phase space volume of the $\mu$ beam by a factor between $10^{5}$ and $10^{6}$. A cascade of recirculating linear accelerators similar to CEBAF rapidly accelerates the $\mu^{+} \mu^{-}$to the collision energy. They are finally stored in a collider ring. The $\mu^{+} \mu^{-}$collisions occur in a fancy low- $\beta$ insertion.

Tab. 1 compares the parameters of a typical proton source for a $\mu^{+} \mu^{-}$collider with the proton source projects KAON ${ }^{11}, \mathrm{JHF}^{12}, \mathrm{ESS}^{13}$, and existing proton synchrotrons. Some of them provide the proton energy $E$, some the repetition frequency $f$, and some the beam power $P$, but none achieves the combination of $E, f$ and $P$ needed for a $\mu^{+} \mu^{-}$collider. 


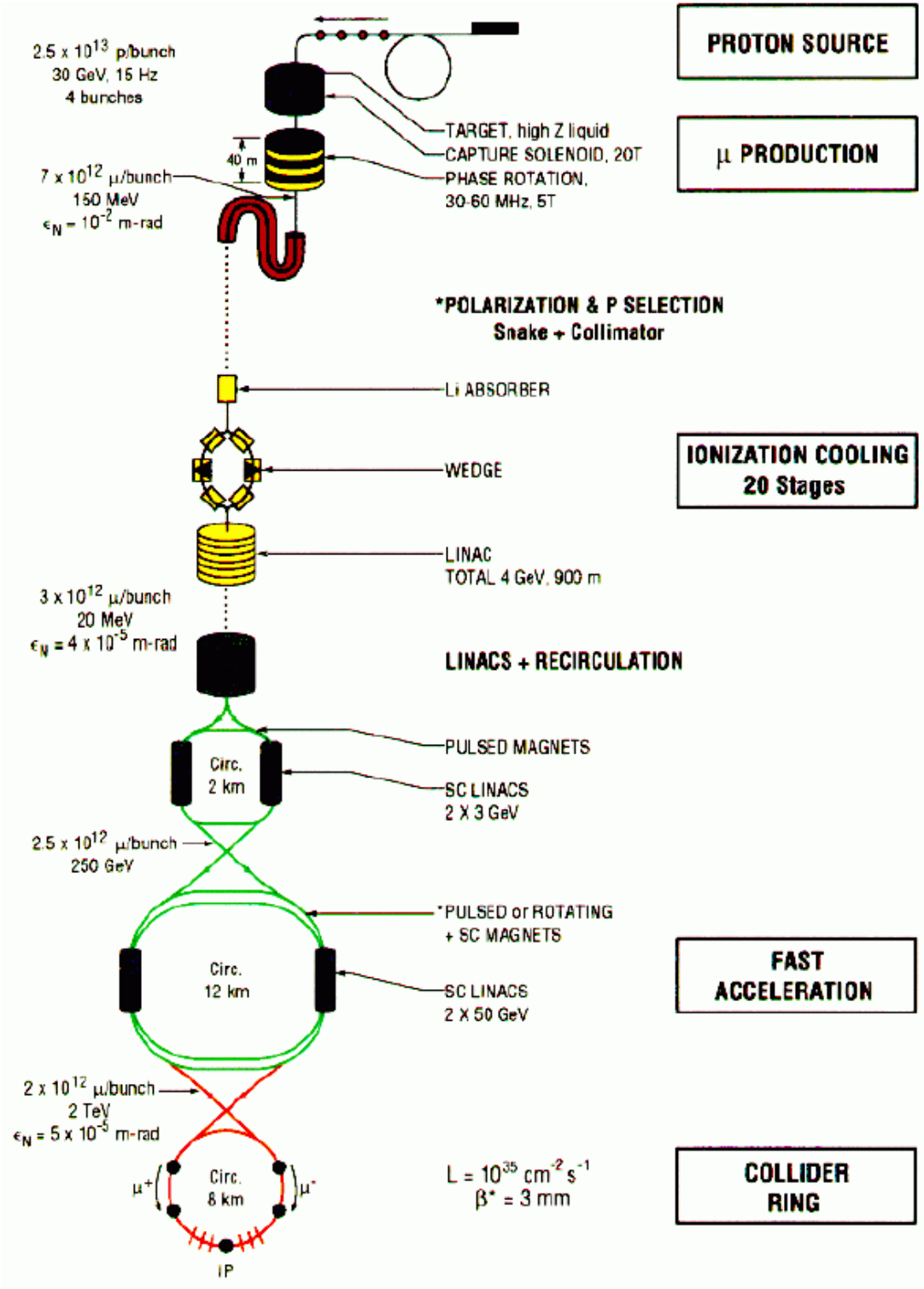

Figure 1: Schematic $4 \mathrm{TeV} \mu^{+} \mu^{-}$Collider: The main components are the proton source, $\mu^{+} \mu^{-}$production, ionization cooling, fast acceleration, and the collider ring. The diagram is not to scale. 
Table 1: Energy $E$, repetition rate $f$ and beam power $P$ of a typical proton synchrotron for $\mu^{+} \mu^{-}$production, and of the KAON, JHF, ESS projects and existing machines

\begin{tabular}{lrrr}
\hline Machine & $E / \mathrm{GeV}$ & $f / \mathrm{Hz}$ & $P / \mathrm{MW}$ \\
\hline$\mu^{+} \mu^{-}$PS & 16 & 15 & 4 \\
KAON & 30 & 10 & 3 \\
JHF & 50 & 0.3 & 0.5 \\
ESS & 1.33 & 50 & 5 \\
FNAL booster & 8 & 15 & 0.067 \\
CPS & 28 & 0.4 & 0.056 \\
\hline
\end{tabular}

\subsection{Muon Collider Ring}

The most important parameters of a $\mu^{+} \mu^{-}$collider ring appear in the following equation for the luminosity $\bar{L}$, averaged over the relativistic $\mu$ lifetime:

$$
\bar{L}=\left(\frac{\tau_{0}}{e \mu_{0}}\right) \dot{N}_{\mu}\left(\frac{\xi \gamma B}{\beta_{\perp}}\right)\left(\frac{2 \pi \rho}{C}\right)
$$

The first bracket contains the natural constants $\mu$ lifetime at rest $\tau_{0}=2.19703 \pm$ $0.00004 \mu \mathrm{s}, \mu$ charge $e$, and permittivity of free space $\mu_{0}$. Then comes the $\mu^{+} \mu^{-}$ storage rate $\dot{N}_{\mu}$, determined by the $\mu^{+} \mu^{-}$source. The second bracket contains the beam-beam tune shift parameter $\xi$, the relativistic factor $\gamma$, the dipole field in the arcs $B$, and the value of the $\beta$-function at the interaction point $\beta_{\perp}$. The last bracket is a filling factor $<1$, the ratio of the total length of dipoles in the arcs $2 \pi \rho$ and the ring circumference $C$. Only two assumptions enter into (1): The interval between fills is long compared to the relativistic $\mu$ lifetime, and the beams are round at the interaction point. For good average luminosity $\bar{L}$, the quantities in the numerator should be large, and those in the denominator small.

Tab. 2 shows the parameters of $\mu^{+} \mu^{-}$colliders at two energies, a high energy one with $3 \mathrm{TeV}$ in the $\mathrm{CoM}$, and a lower energy "Higgs factory" at $100 \mathrm{GeV}$ in the CoM. Because of their larger mass, $\mu$ 's produce much less synchrotron radiation than electrons of the same energy, and can be recirculated and stored in circular machines at high bending field. Hence, $\mu^{+} \mu^{-}$colliders are much more compact than circular $\mathrm{e}^{+} \mathrm{e}^{-}$colliders at the same energy. The $3 \mathrm{TeV} \mu^{+} \mu^{-}$collider has about the size of the SPS at CERN with $C=6911 \mathrm{~m}$, while the $\mathrm{e}^{+} \mathrm{e}^{-}$collider LEP at CERN with $200 \mathrm{GeV}$ in the CoM has $C=26659 \mathrm{~m}$. The arcs of a $\mu^{+} \mu^{-}$collider have a high dipole field in order to maximise the number of turns in the $\mu^{+} \mu^{-}$lifetime. They are nearlyisochronous in order to achieve short bunches. The low- $\beta$ insertion uses techniques from final-focus systems of linear $\mathrm{e}^{+} \mathrm{e}^{-}$colliders to achieve a low value of $\beta_{\perp}$ at the 
Table 2: Parameters of $\mu^{+} \mu^{-}$colliders

\begin{tabular}{|l|c|cc|}
\hline CoM Energy $(\mathrm{GeV})$ & 3000 & \multicolumn{2}{|c|}{100} \\
\hline No. of $\mu^{ \pm}$bunches/sign & 2 & \multicolumn{2}{|c|}{1} \\
Bunch population $\left(10^{12}\right)$ & 2 & \multicolumn{2}{|c|}{4} \\
Collider circumference $(\mathrm{m})$ & 6000 & \multicolumn{2}{|c|}{300} \\
\hline RMS momentum spread $(\%)$ & 0.16 & 0.12 & 0.003 \\
\hline Free space $\ell^{*}$ at IP $(\mathrm{m})$ & 6.5 & \multicolumn{2}{|c|}{5} \\
$\beta^{*}$ at IP $(\mathrm{cm})$ & 0.3 & 4 & 13 \\
Bunch length $\sigma_{z}(\mathrm{~cm})$ & 0.3 & 4 & 13 \\
Beam radius at IP $(\mu \mathrm{m})$ & 3.2 & 82 & 270 \\
Beam-beam tune shift $\xi$ & 0.043 & 0.05 & 0.015 \\
\hline Luminosity $(\mathrm{nbs})^{-1}$ & 50 & 0.12 & 0.01 \\
\hline
\end{tabular}

interaction point IP where the detector is installed. The projected beam-beam tune shift $\xi$ is higher than in hadron colliders, and comparable to that of $\mathrm{e}^{+} \mathrm{e}^{-}$colliders.

\subsection{Critical $\mu^{+} \mu^{-}$Collider Issues}

Many of the components of a $\mu^{+} \mu^{-}$collider system are at the limit of what is technologically possible. These components should be tested one by one. A proposal for an initial cooling experiment was submitted to Fermilab ${ }^{15}$. A proposal for a targetting experiment at BNL is being prepared.

Collimated neutrino beams from muon decay in straight section are a radiation hazard, imposing upper limit on muon energy and lower limit on depth of $\mu^{+} \mu^{-}$ collider.

\section{Future Larger Hadron Colliders}

Future larger hadron colliders beyond the LHC and the discontinued SSC were discussed in 33 Eloisatron studies at Erice ${ }^{16}$ since 1986, and in 'VLHC' workshops at Indianapolis and Snowmass ${ }^{17}$ since 1996. Exploratory studies continue in several laboratories ${ }^{18,19,20}$. A National vlhc Organization was recently set up in the US with a steering committee, working groups, meetings, etc. ${ }^{21}$.

\subsection{FLHC Studies}

Tab. 3 compares the parameters of the LHC with those of three larger machines, a $50 \mathrm{TeV}$ collider $\mathrm{LoB}$ with $1.8 \mathrm{~T}$ dipoles ${ }^{17}$, a $50 \mathrm{TeV}$ collider HiB with $12.6 \mathrm{~T}$ dipoles $^{17}$, and a $100 \mathrm{TeV}$ collider with $12 \mathrm{~T}$ dipoles ${ }^{16}$. The combination of a high 
and a low dipole field $B$, and of two energies $E$, and the comparison with the LHC, clearly show how the choice of $B$ and $E$ changes the parameters. The stored energy is given in tons of TNT. The conversion factor is $1 \mathrm{t} \mathrm{TNT} \equiv 4.7$ GJ. The LoB collider has combined-function arcs. Hence, it is anti-damped, and the damping time is negative, but long enough.

Table 3: Comparison of LHC and FLHC Parameters

\begin{tabular}{|c|c|c|c|c|}
\hline & LHC & LoB & $\mathrm{HiB}$ & $\mathrm{E} 12 \mathrm{~T}$ \\
\hline Beam energy/TeV & 7 & 50 & 50 & 100 \\
\hline Dipole field/T & 8.4 & 1.8 & 12.6 & 12 \\
\hline Circumference/km & 27 & 646 & 104 & 229 \\
\hline Luminosity/(nbs) $)^{-1}$ & 10 & 10 & 12 & 10 \\
\hline Bunch spacing/ns & 25 & 16.7 & 16.7 & 37.5 \\
\hline Events/collision & 19 & 10 & 12 & 23 \\
\hline Vert. damping time/h & 26 & - ve & 2.6 & 1.5 \\
\hline Radiation power/kW & 3.7 & 48 & 189 & 1080 \\
\hline Stored energy/t TNT & 0.07 & 2.07 & 0.19 & 0.63 \\
\hline Debris power/kW & 0.8 & 4.8 & 5.8 & 9.6 \\
\hline
\end{tabular}

Contrary to a $\mu^{+} \mu^{-}$collider system, the scale of an FLHC system is dominated by the collider ring proper, not by its injectors. The number of events in a collision, $n_{c}=L \sigma_{\text {inel }} s$, expresses the ease or difficulty of analyzing the events in the detector, with inelastic cross section $\sigma_{\text {inel }}$. Keeping it at values comparable to those for LHC essentially imposes an upper limit on the bunch spacing $s$. The power in the debris $D=L \sigma_{\text {inel }} E$ of the collisions, which must be absorbed by shielding to prevent the cascades from heating super-conducting coils, is given by the performance parameters, leaving no choice for the designer.

\subsection{Critical FLHC Issues}

The product of damping time $\tau_{z}$ and damping partition number $J_{z}$ for synchrotron radiation in the arbitrary $z$-plane is:

$$
\tau_{x} J_{x}=\left(\frac{3 E_{0}^{3}}{e^{2} c^{3} r_{c}}\right) \frac{1}{E B^{2}}\left(\frac{C}{2 \pi \rho}\right)
$$

Here $E_{0}$ is the rest mass of the particle and $r_{c}$ its classical radius. Numerically the factor in the left bracket is $16644 \mathrm{~h} \mathrm{TeV} \mathrm{T} \mathrm{T}^{2}$. The right bracket is the reciprocal of the filling factor appearing in (1). By using (2), the synchrotron radiation power $P$, that may cause a considerable heat load when it gets absorbed in a vacuum chamber at 
cryogenic temperatures, and the stored energy in one beam $G$, that must be absorbed by a beam dump without destroying it, can be brought into the following forms:

$$
\begin{gathered}
P=\left(\frac{4 \pi r_{c}^{3 / 2}}{\sqrt{3 c E_{0}}}\right) \frac{E^{3 / 2} L \beta_{\mathrm{IP}}}{\xi \sqrt{J_{z} \tau_{z}}} \sqrt{\frac{C}{2 \pi \rho}} \\
G=\left(\frac{2 \pi r_{c}^{3 / 2}}{\sqrt{3 c E_{0}}}\right) \frac{E^{3 / 2} L \beta_{\mathrm{IP}} \sqrt{J_{z} \tau_{z}}}{\xi} \sqrt{\frac{C}{2 \pi \rho}}
\end{gathered}
$$

The central fraction in (3) and (4) contains the design parameters $E, L, \beta_{\mathrm{IP}}$, and $\xi$, and opposite powers of $\sqrt{J_{z} \tau_{z}}$. By choosing $B$, and hence $\tau_{z}$, one can trade a reduction of $P$ against and increase of $G$, and vice-versa.

An important collective effect is the transverse resistive-wall instability ${ }^{20}$. Its growth rate is a function of the conductivity, and hence depends on the composition and temperature of the vacuum chamber. By choosing the temperature, one can trade the growth rate of the instability and the feedback system needed to damp it against the heat load caused by the synchrotron radiation absorbed by the vacuum chamber. A second important collective effect is the coherent synchrotron tune shift, driven by the longitudinal broad-band impedance ${ }^{20}$. In order to ensure longitudinal Landau damping, the bunch length must be increased. In the high $B$ colliders, the growth times caused by intra-beam scattering are only a little larger than the damping times ${ }^{20}$.

Simply scaling the FLHC cost from LEP, LHC and/or SSC would result in exorbitant figures. Therefore, $R \& D$ programmes, aiming at significant reductions of unit prices, have been launched. In the US, they are coordinated by the National vlhc Organization. Figs. 2 and 3 show examples of magnet designs at LBNL ${ }^{22}$ and Fermilab $^{23}$.

1. G.I. Budker, Accelerators and Colliding Beams, Proc. 7th Internat. Accelerator Conference (Erevan, 1969) Vol. I, 33, Talk at the International High Energy Physics Conference (Kiev, 1970).

2. V.V. Parkhomchuk and A. N. Skrinsky, Proc. 12th Int. Conf. on High Energy Accelerators, F. T. Cole and R. Donaldson (Eds.) (1983) 485; A. N. Skrinsky and V.V. Parkhomchuk, Sov. J. Part. Nucl. 12 (1981) 223; D. Cline, AIP Conf. Proc. 352 (1996) 3.

3. D. Neuffer, IEEE Trans. NS-28, (1981) 2034.

4. D. Cline, Nucl Inst. and Meth. A350 (1994) 24-56; Proc. of the Muon Collider Workshop, February 22, 1993, Los Alamos National Laboratory Report LAUR-93-866 (1993); Physics Potential \& Development of $\mu^{+} \mu^{-}$Colliders $2^{\text {nd }}$ Workshop, Sausalito, California, D. Cline (Ed.), AIP Conf. Proc. 352 (1996).

5. Transparencies at the $2+2 \mathrm{TeV} \mu^{+} \mu^{-}$Collider Collaboration Meeting, Feb 68, 1995, BNL, compiled by Juan C. Gallardo; www. cap.bnl . gov/mumu/ 
Transparencies at the $2+2 \mathrm{TeV} \mu^{+} \mu^{-}$Collider Collaboration Meeting, July 11-13, 1995, Fermilab, compiled by R. Noble; Proc. of the 9th Advanced ICFA Beam Dynamics Workshop, Ed. J. C. Gallardo, AIP Conf. Proc. 372 (1996).

6. D.V. Neuffer and R.B. Palmer, Proc. EPAC'94 (London, 1994) 52; M. Tigner, AIP Conf. Proc. 279 (1993) 1.

7. R.B. Palmer et al., AIP Conf. Proc. 352 (1996) 108;

R. B. Palmer et al., Nucl. Phys. B (Proc. Suppl.) 51A (1996).

8. $\mu^{+} \mu^{-}$Collider: A Feasibility Study, BNL-52503, Fermilab-Conf-96/092, LBNL-38946 (1996).

9. R.B. Palmer, A. Tollestrup and A. Sessler, New Directions for High-Energy Physics (Snowmass, 1996) 203.

10. Muon Collider Collaboration, Status of Muon Colliders and Future Research and Development Plans, to be published,

cf. www.cap.bnl.gov/mumu/status_report.html.

11. KAON Factory Study-Accelerator Design Report, TRIUMF, Vancouver B.C., Canada (1990).

12. Y. Mori et al., Proc. EPAC'96 (Sitges, 1996) 569.

13. H. Lengeler et al. (ed.), The European Spallation Source, Vol.III, The ESS Technical Study, ESS-96-53-M (1996).

14. R.B. Palmer, Talk presented at the 3rd meeting of the HEPAP Subpanel on Planning for the Future of U.S. High Energy Physics, 12-14 August, 1997.

15. www. fnal.gov/projects/muon_collider/

16. A.G. Ruggiero (ed.), Hadron Colliders at the Highest Energy and Luminosity (World Scientific, Singapore 1998).

17. G. Dugan, P. Limon, M. Syphers, Proc. New Directions for High-Energy Physics (Snowmass, 1996) 251.

18. www-ap. fnal.gov/VLHC.

19. S. Peggs, M. Harrison, F. Pilat, M. Syphers, Proc. PAC'97 (Vancouver 1998) 95.

20. E. Keil, Proc. PAC'97 (Vancouver 1998) 104.

21. www. vlhc.org.

22. R. Gupta, Proc. PAC'97 (Vancouver 1998) 3344.

23. G.W. Foster et al., Proc. PAC'97 (Vancouver 1998) 3392. 


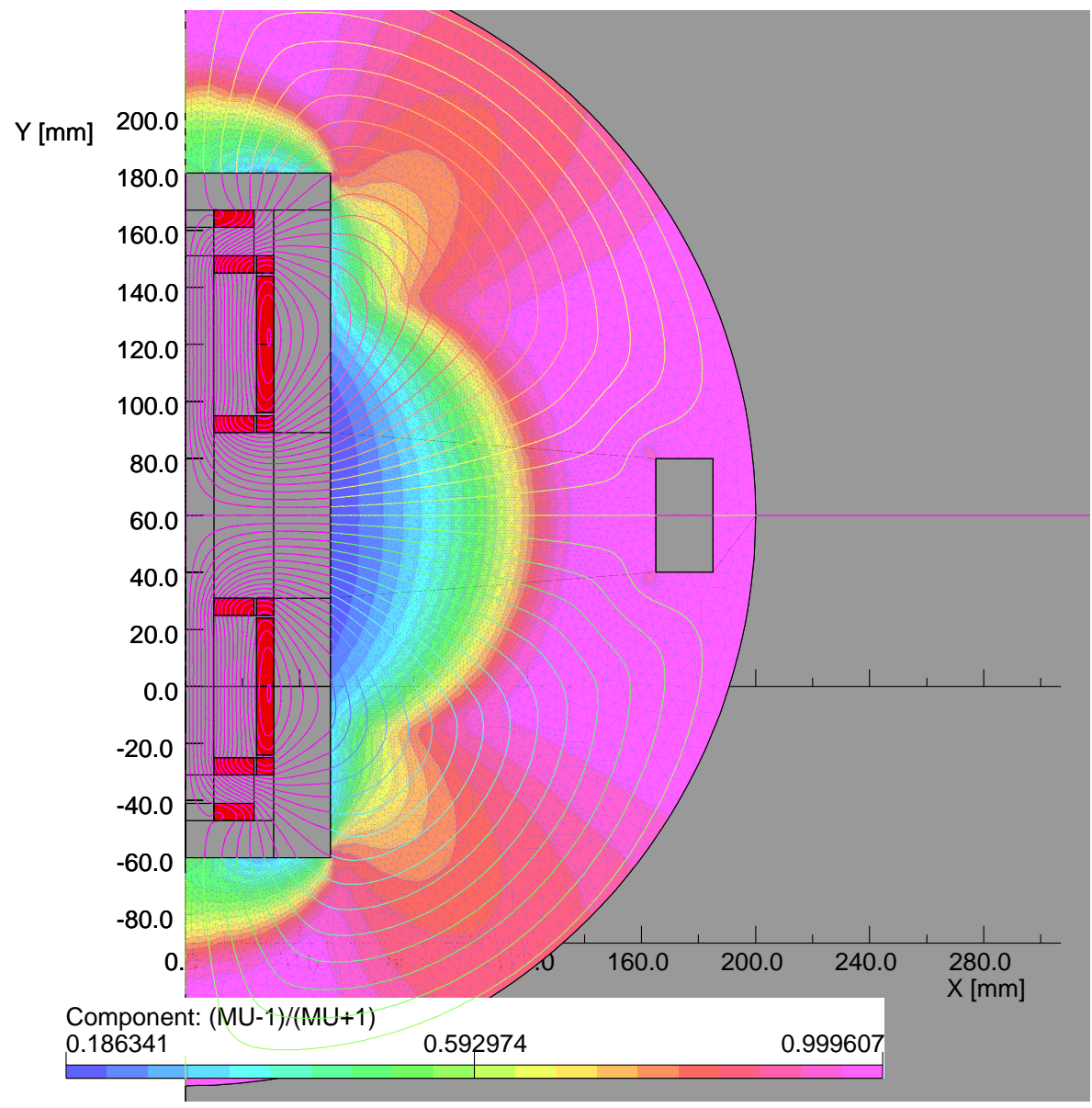

Figure 2: Concept of Half a High $B$ Dipole at LBNL: The coils and beam apertures are in the upper and lower part of the the rectangular region to the left. The half circle is the steel yoke with magnetic field lines. The colour code shows its saturation. 


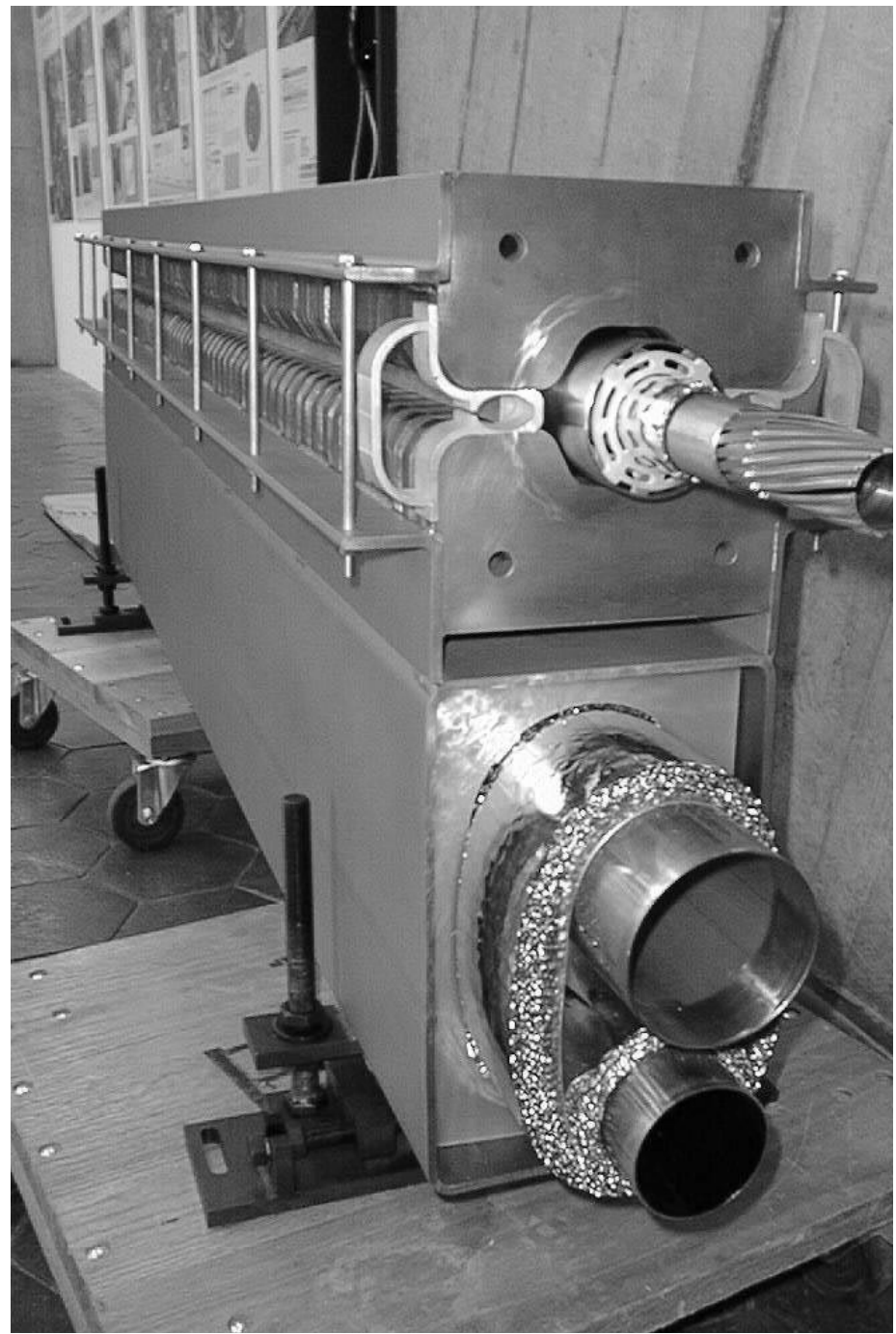

Figure 3: Model of Low $B$ Dipole at Fermilab: The upper part shows the dipole proper with the left extruded Al beam pipe and the super-conducting $75 \mathrm{kA}$ transmission line. The lower part shows the structural support tube, the cryoline vacuum jacket, the current return line, and the cryogenic piping for the distribution of single-phase He along the ring. 